\title{
Vibration based condition monitoring of rotating machinery
}

\author{
Goutam Senapaty ${ }^{1 *}$ and Sathish Rao U. ${ }^{1}$ \\ ${ }^{1}$ Department of Mechanical and Manufacturing Engineering, Manipal Institute of Technology, \\ Manipal Academy of Higher Education, Manipal, India
}

\begin{abstract}
This project looks at the different maintenance philosophies and the importance of vibration analysis in predictive maintenance. Since most industries and plants make use of rotational equipment, vibration analysis plays a major role in detecting machine defects and developing flaws before the equipment fails and potentially damages other related equipment and to avoid unwanted breakdowns and downtime. Vibration analysis can help increase the lifetime of equipment when the faults are diagnosed at the right time. Vibration analysis of a rotating table top model is also done to show that some faults might exist even though they are not visible to the naked eye.
\end{abstract}

\section{Maintenance Philosophies and the role of Vibration Analysis}

Plant maintenance is one of the most important aspects of running a plant or an industry, as the maintenance philosophy a plant follows will determine how much downtime the plant has to go through and the profit margin of the plant. Money saved in maintenance is a direct contribution to improved plant profitability. The main goal of maintenance is to minimize scheduled and unscheduled downtime, improve safety and maximize the availability of production equipment.

The common maintenance philosophies followed by plants and industries are as follows

- Reactive maintenance: This type of maintenance is also known as Break-down or On-failure maintenance. This type of maintenance is done during shutdowns after the failure of the equipment. Due to the nature of this philosophy, there is no warning of failure; there are uncontrolled plant outages and production losses or delays and a large inventory of spare parts I required.

- Preventive maintenance: This type of maintenance is also known as Fixed-time or time-based maintenance. Maintenance of equipment is based on a fixed time calendar based, hours run based or number of equipment cycles based. This maintenance philosophy helps reduce failures, allows work, spare parts and labour to be planned. Despite its advantages, preventive maintenance is more costly and will cause frequent downtimes due to the nature of the maintenance, so it is only applicable to machines that undergo age related deterioration, otherwise it may lead to unnecessary replacement of part and components.

\footnotetext{
*Corresponding author: goutam.senapaty@gmail.com
} 
- Proactive Maintenance: This type of maintenance is also known as predictive maintenance or condition based maintenance. This maintenance philosophy is very advantageous to the plant as machine faults can be detected before failure occurs, without doing regular scheduled maintenance. This maintenance philosophy requires regular and frequent monitoring of the machine health condition. Practices such vibration analysis, infrared thermography, ultrasound and various other forms of non-destructive testing can be used to detect deterioration of machine health and problems in the components before they fail and require the plant to be shut down. Usually critical plant equipment undergo this form of philosophy. It helps in reducing downtime, detecting root cause of the failure and managing resources such as labour and spare parts effectively.

Proactive maintenance philosophy is the best way to manage and maintain plant most the components and equipment in a plant or industry, but only if used effectively and in required places, otherwise it can get very expensive. It is important to find out defects with the equipment before they fail so that their failure doesn't affect the condition of equipment that are working with them and further cause more damage to the plant.

\subsection{Why Vibration}

Since the beginning of building machines, especially rotating machines, reduction and isolation of vibration has remained to be a key area of focus. Gradually as vibration reduction and isolation techniques have become more and more common, the need for accurate measurement of vibration has also become increasingly popular. Within the last 15-20 years, modern vibration measuring equipment have been developed for investigating high-load, high-speed machinery.

\subsection{Vibration Characteristics}

Amplitude - Helps in detecting the severity of the fault condition or defect.

Frequency - Helps in indicating the cause of the defect

Phase - Helps in determining the cause of the defect

Vibration amplitude can be measured in terms of displacement, velocity, acceleration. Displacement is measured peak to peak and usually in mils or microns. Velocity can be measured in peak or RMS and usually in inch/sec or $\mathrm{mm} / \mathrm{sec}$. Acceleration is expressed in peak and usually measured in g's. Phase indicates how a machine is moving to a reference of its part. The relationship of the movement of part of a machine to a reference - for example the position of both end of the shaft as it rotates.

This indicates the relationship of the movement between one or more points on a machine. 


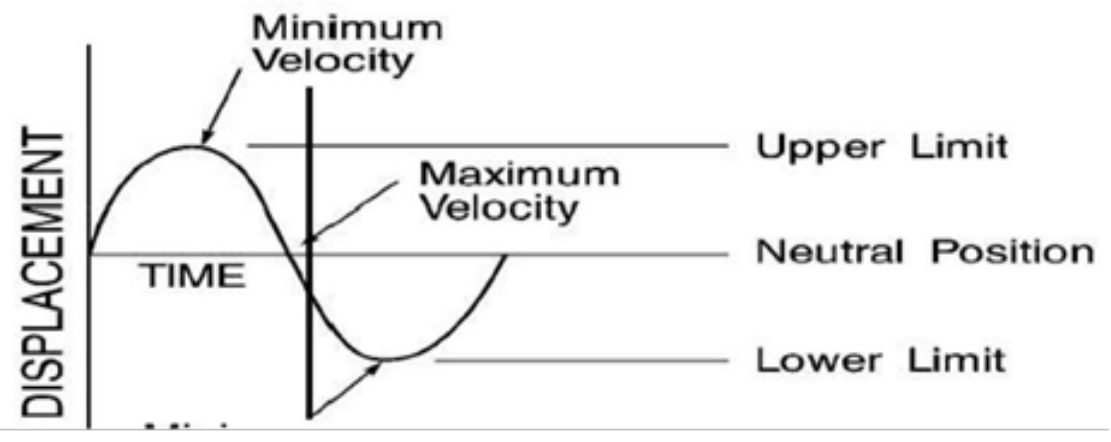

Fig. 1. Time waveform of a simple harmonic motion [1]

\section{How to study and analyse vibration data}

\subsection{Quantifying the vibration level}

The vibration amplitude, which describes the severity of the vibration, can be quantified in several ways. The Peak-to-Peak value indicates the maximum excursion of the wave, a useful quantity where, for example, the vibratory displacement of a machine part is critical for maximum stress or mechanical clearance considerations.

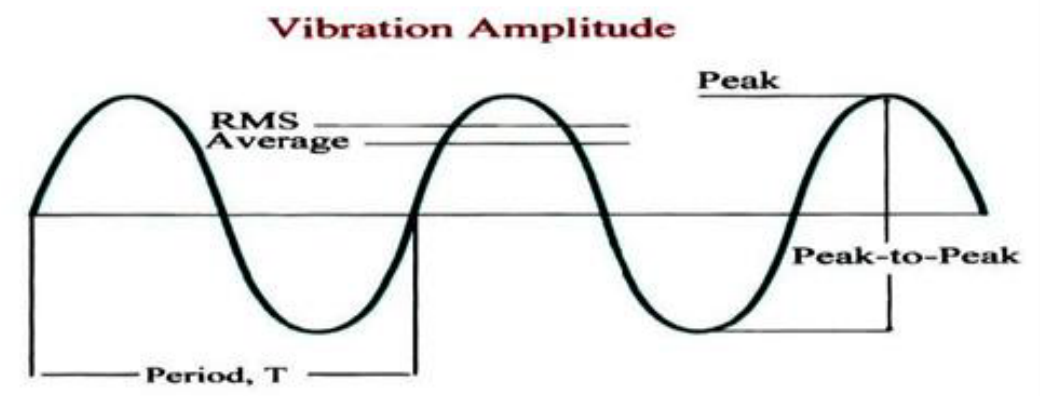

Fig. 2. Peak and RMS values [1] 


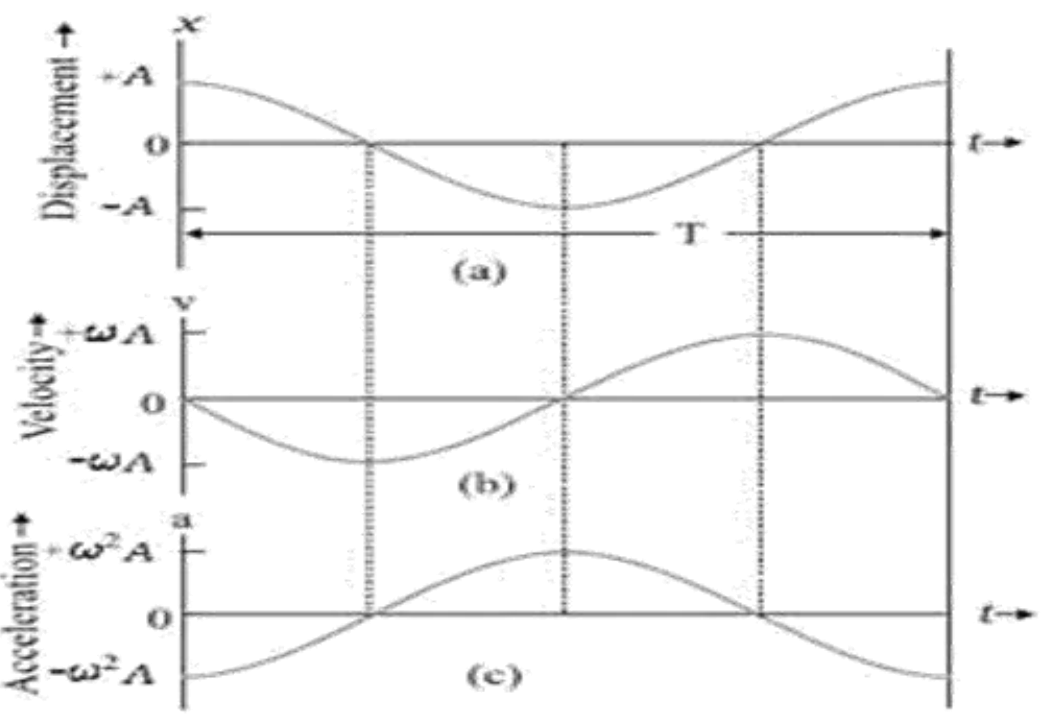

Fig. 3. Displacement, Velocity and Acceleration graphs [1]

\section{Transducer Effectiveness Regions}

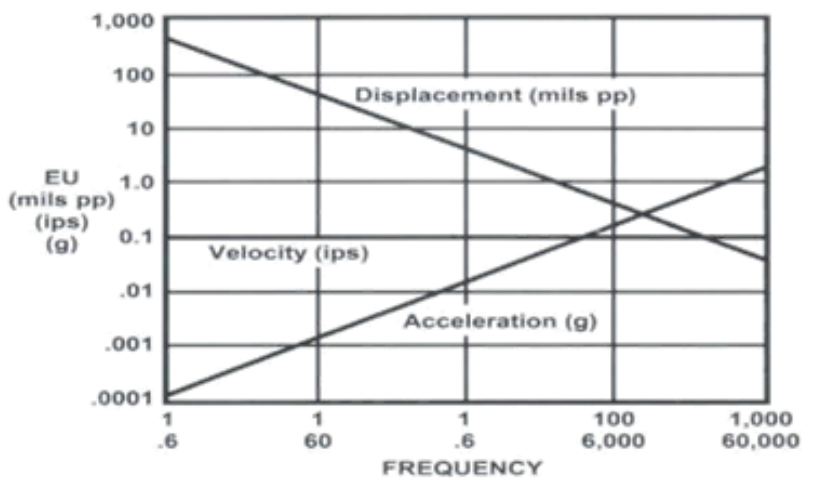

\section{Displacement: \\ When the speed is less than 1000 CPM and the machine has journal bearings \\ Velocity: \\ When the speed is between 600 CPM and 120,000 CPM}

\section{Acceleration:}

When the speed is greater than 60,000 CPM

Fig. 4. Transducer effectiveness regions [1]

\subsection{What are $F_{\max }$, Lines and Averages?}

\subsubsection{Guidelines for setting $F_{\max }$ (End Frequency)}

- For machines with Rolling Element Bearings: $F_{\max }=60 \times$ RPM.

- For machines with Journal / Sleeve Bearings: $F_{\max }=20 x$ RPM.

- For machines with Gear Box : $F_{\max }=200 \times$ RPM. 


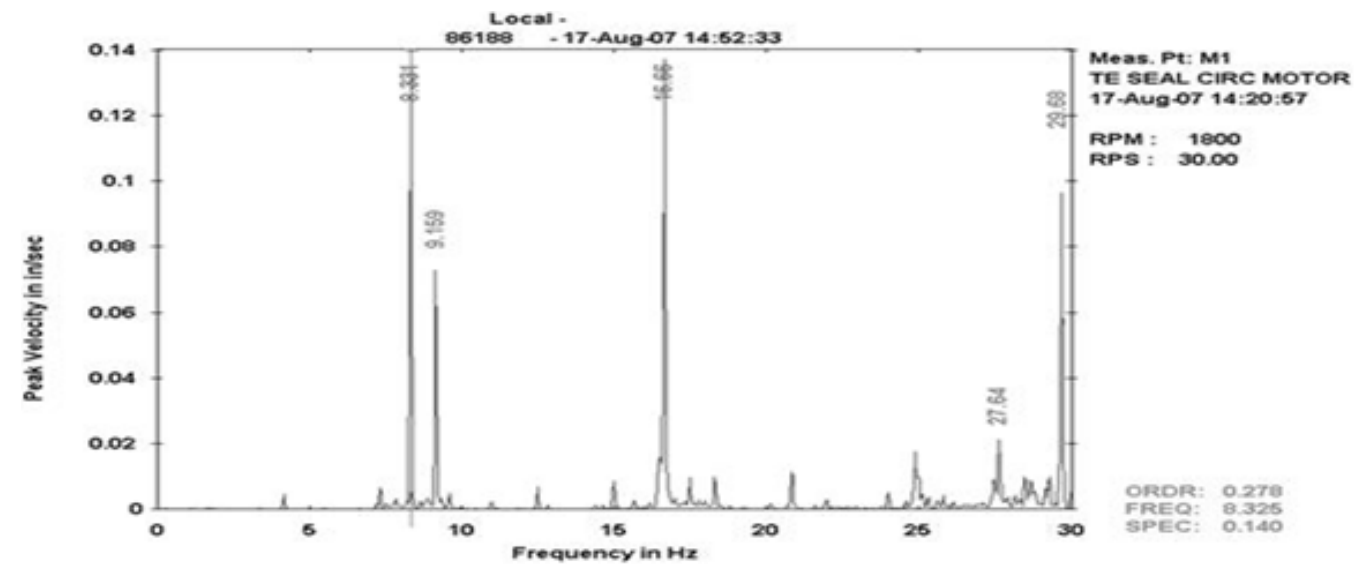

Fig. 5. Example of insufficient $F_{\max }[1]$

\subsubsection{Guidelines for setting Number of Lines (Resolution)}

- Number of lines determines the Machine spectrum resolution.

- $\quad$ Band width $(\mathrm{BW})=\mathrm{F}_{\max } /$ No. of Lines.

- More the lines, less is the bandwidth \& hence higher the resolution \& better the accuracy.

- Typically, 400, 800 or 1600 lines are used, as more the lines more the resolution.

\subsubsection{Number of Signal Averaging}

- Averaging is used to reduce random content in the vibration signal.

- Typically, 2-4 averages are used.

- Again, more the averages, more is the data collection time.

\subsection{Time Waveform Analysis}

Time waveform is the raw data generally acquired by the analyser and also plays a vital role in the analysis process. The amplitude units and the acceleration peak to peak values will be the initial focus for the analyst. The amplitude reflects the severity of the vibration against time domain. This is also known as the machine raw data, as it vibrates while running. The time waveform contains very important information. Although many analysts do not view the time wave form during data analysis, it should be noted that the waveform data is the source that is used to generate the spectral data and trend data that are commonly used for fault detection. 


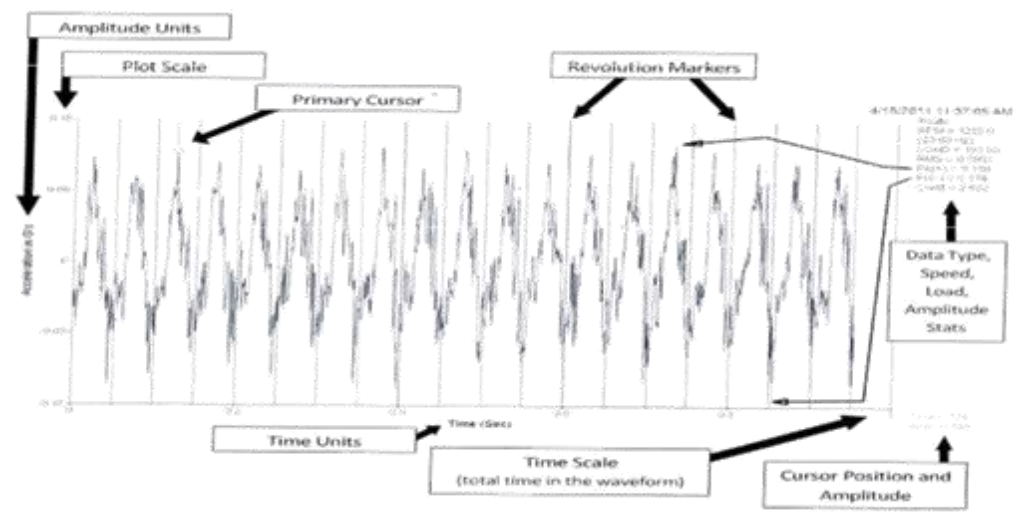

Fig. 6. Example of Time Waveform [1]

The length of the waveform should be long enough to include 10 to 15 shaft rotations.

\subsection{What is FFT?}

FFT stands for "Fast Fourier Transform". It is possible to take a vibration time waveform, whether simple or complex, and mathematically calculate the vibration frequencies present along with their amplitudes with the help of FFT.

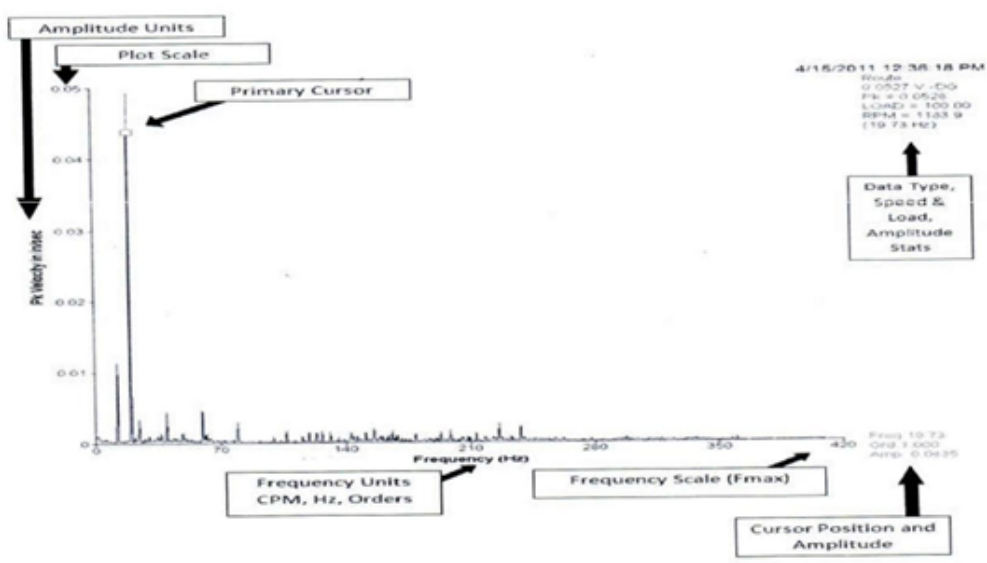

Fig. 7. Velocity Spectrum [1]

Spectrum analysis is the procedure of doing the transformation, and is invariably done with the help of FFT analyser. Signature term usually applied to the vibration frequency spectrum which is unique to a machine at a specific point in time, under specific machine operating conditions.

Normal Frequencies: Vibration peaks that appear in the spectrum as a normal condition of the function of the machine such as running speed, fan blade pass or pump vane pass etc. 
Defect (fault) Frequencies: Calculated frequencies that appear in the spectrum only when there is a problem with the component such as a bearing defect frequency.

Fundamental Frequency: It is also called the first harmonic. Usually it's a pre-calculated frequency, such as turning speed of the machine or a frequency that is equal to a defect in the machine.

Dominant Peak: A peak in the spectrum with the highest amplitude value or a sharp maximum

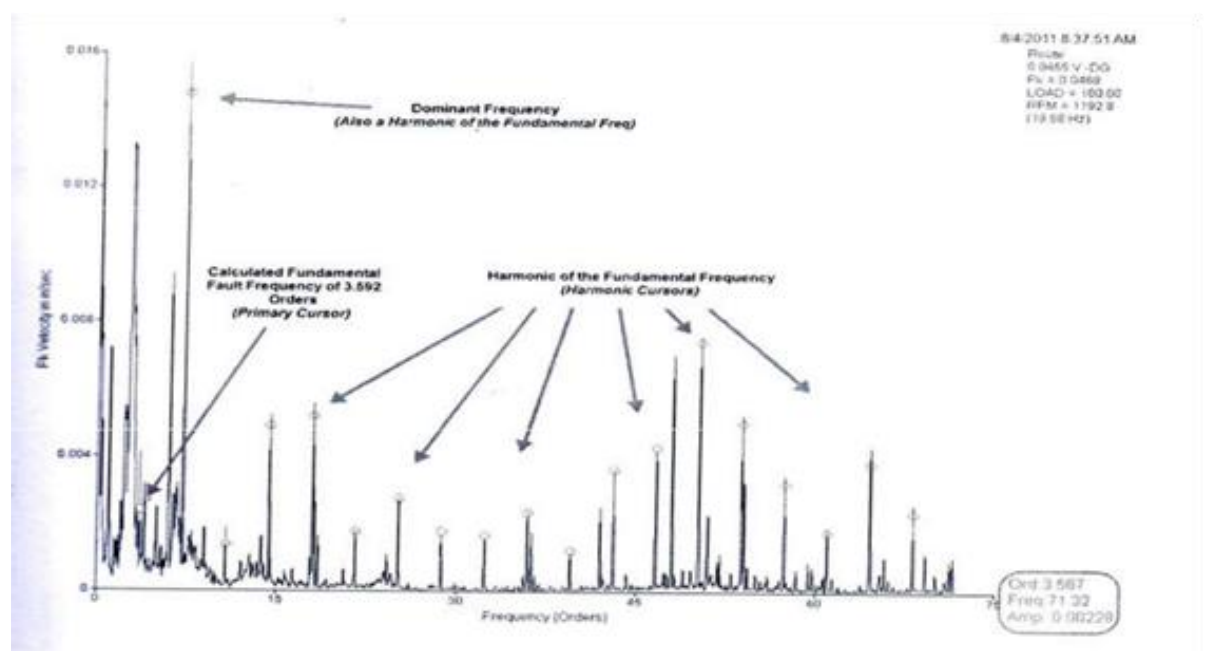

Fig. 8. Velocity Spectrum showing dominant peak [1]

Harmonics: Spectral peaks that occur at integer (whole number) multiples of any frequency. It is possible to have a spectrum where the fundamental is not visible but the harmonics will be spaced apart by the fundamental frequency.

Sub-harmonic: Frequency that is integral sub-multiple of a fundamental frequency.

Synchronous: These are spectral components which are integer multiples or harmonics of a fundamental frequency. They may be present as multiples of an integral fraction, which are called sub-harmonics. 


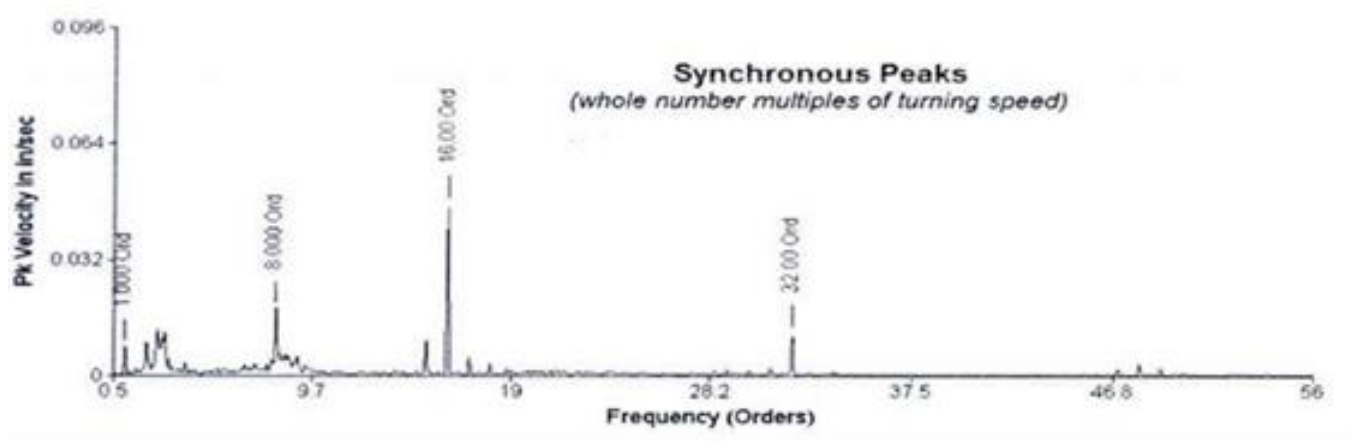

Fig. 9. Synchronous Peaks [1]

Sub-synchronous: Components of a vibration signal which has a frequency, less than the shaft rotation frequency

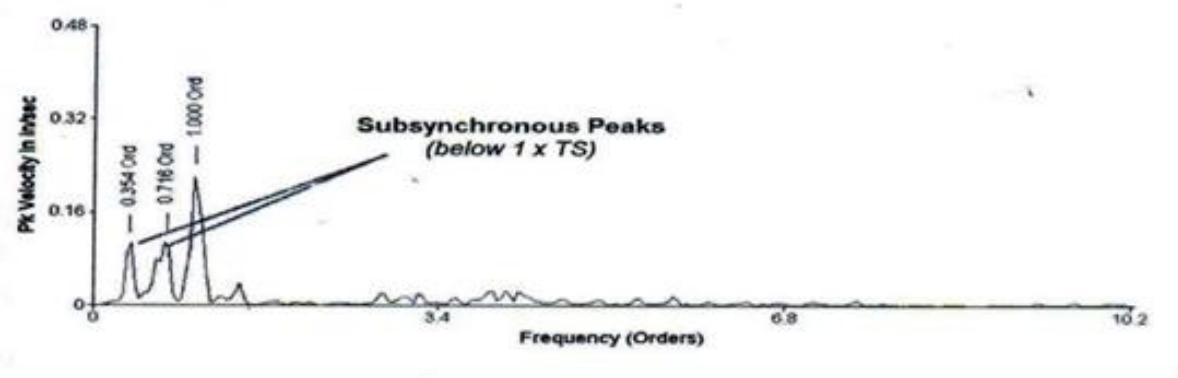

Fig. 10. Sub-Synchronous Peaks [1]

Nonsynchronous Spectra: Spectral peaks that are above turning speed, but are not whole number multiples of turning speed

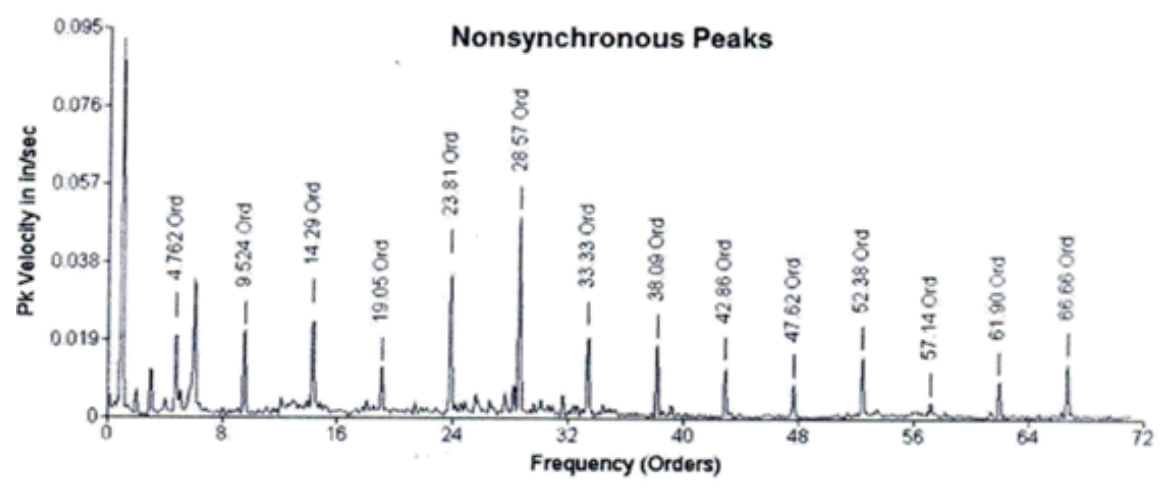

Fig. 11. Non-Synchronous Peaks [1] 
Sidebands: A pattern of equally spaced peaks above and below a centre calculated frequency. Sidebands are caused by modulation of a carrier frequency.

Carrier (centre) Frequency: A vibration peak that is either changing in amplitude or frequency. The carrier frequency is the "centre" frequency that appears between the sideband peaks.

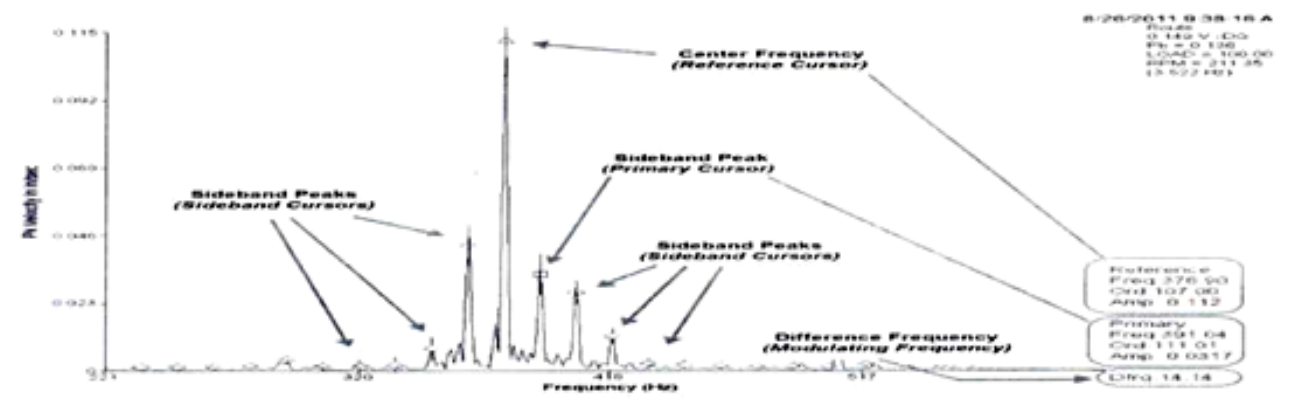

Fig. 12. Vibration spectra showing carrier frequency [1]

\section{Performing vibration analysis on a table top model}

A Table top model consisting of a variable speed motor (mainly used for a sewing machine) of 145 Watt was used to rotate the shaft holding a disc of weight of 500 grams, supported by two anti-friction bearings on either side of the disc. The input shaft is connected to the output shaft using a 3 - jaw coupling.

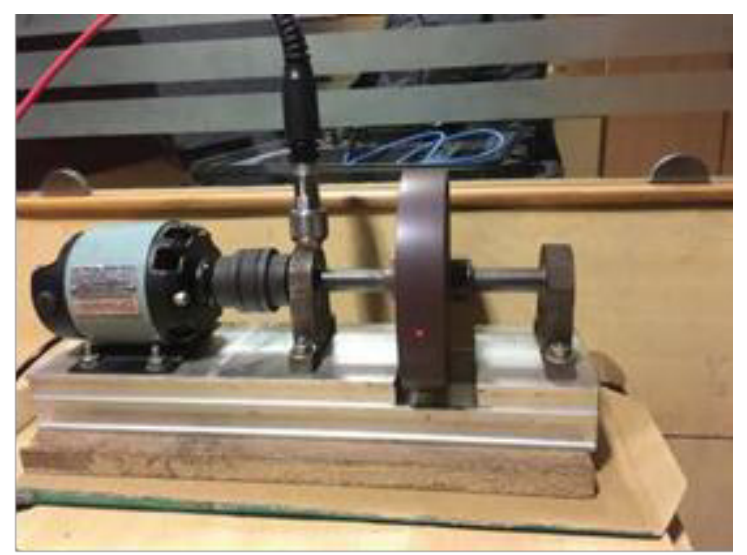

Fig. 13. Table top rotating equipment

A laser tacho-probe was used to determine the speed of rotation and the phase. The tachoprobe is used to measure the speed of rotation with the help of reflective tape which was placed on the disc, it determines speed by recording the time elapsed between the first reflected laser beam and the second time when beam is reflected during the rotation. 


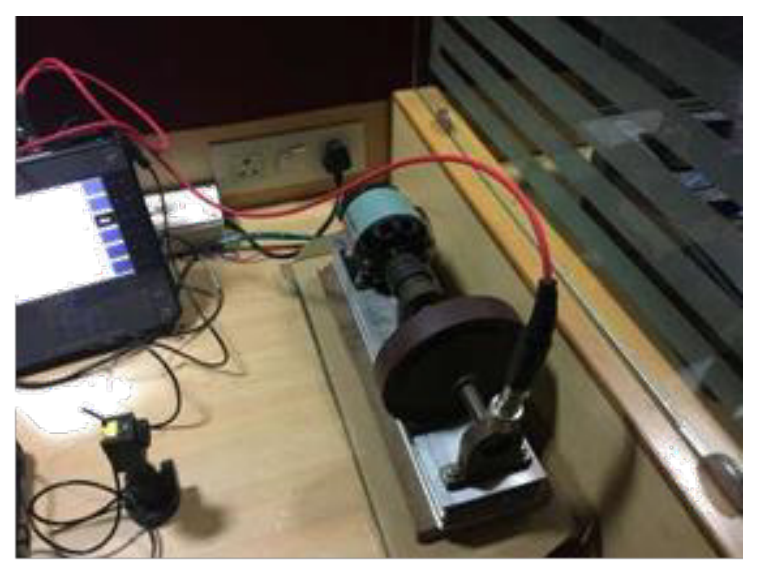

Fig. 14. Table top model with laser tacho-probe and accelerometer

Vibration of the model was recorded at 3 different spots, Motor outboard, Rotor inboard and Rotor outboard. Usually, 3 readings, namely axial, horizontal and vertical readings are taken at each of these positions, but it was not possible to take axial and horizontal reading on motor outboard and rotor inboard due to design limitation of the model and size limitations of the Accelerometer probe.

\subsection{Methodology}

1. First, speed is measured using the tacho-probe. The required cable attachments are made to the CSI 2140, the tacho-probe is placed parallel to the disc on which reflective tape is put, at a distance such that it is not too close, but not too far, such that the beam of laser is no wider than a point.

2. After speed is determined, the accelerometer cable is attached to the probe and to the machine health analyser and then placed on suitable respective points on the model, usually on the bearing housings.

3. Adjustments are made to the model to align it properly, then above steps are repeated again.

4. For single plane balancing,

o Create new job

o Set up job parameters (decide which plane to add/remove weights)

o Acquire reference run data

o Add trial weight

o Acquire trial run data

o View the balance correction 
o Remove trial weights and add/remove correction weight

o Collect trim run data for corrected condition

\subsection{Data collection and results obtained}

1. First the monitoring locations (Six) for the Table top model was created in the computer interface of the analyser and then uploaded to the CSI 2140 analyser for collecting the data with the help of accelerometer for one of the locations.

2. All the collected data from the analyser was downloaded to the computer interface software and is viewed, showing the location Overall values and the spectra before and after the Alignment.

3. During Analysis in given state, model showed characteristics of having misaligned shaft, which was found out due to occurrence of $3 x$ peaks in all the six locations

4. Readings after proper alignment of coupling was taken, and the shaft was known to be aligned as the $3 \mathrm{x}$ peaks had disappeared.

PKS It:m/ 101 / TABLE TOP MOOEL / MOV - Motor Outboard Vertocal
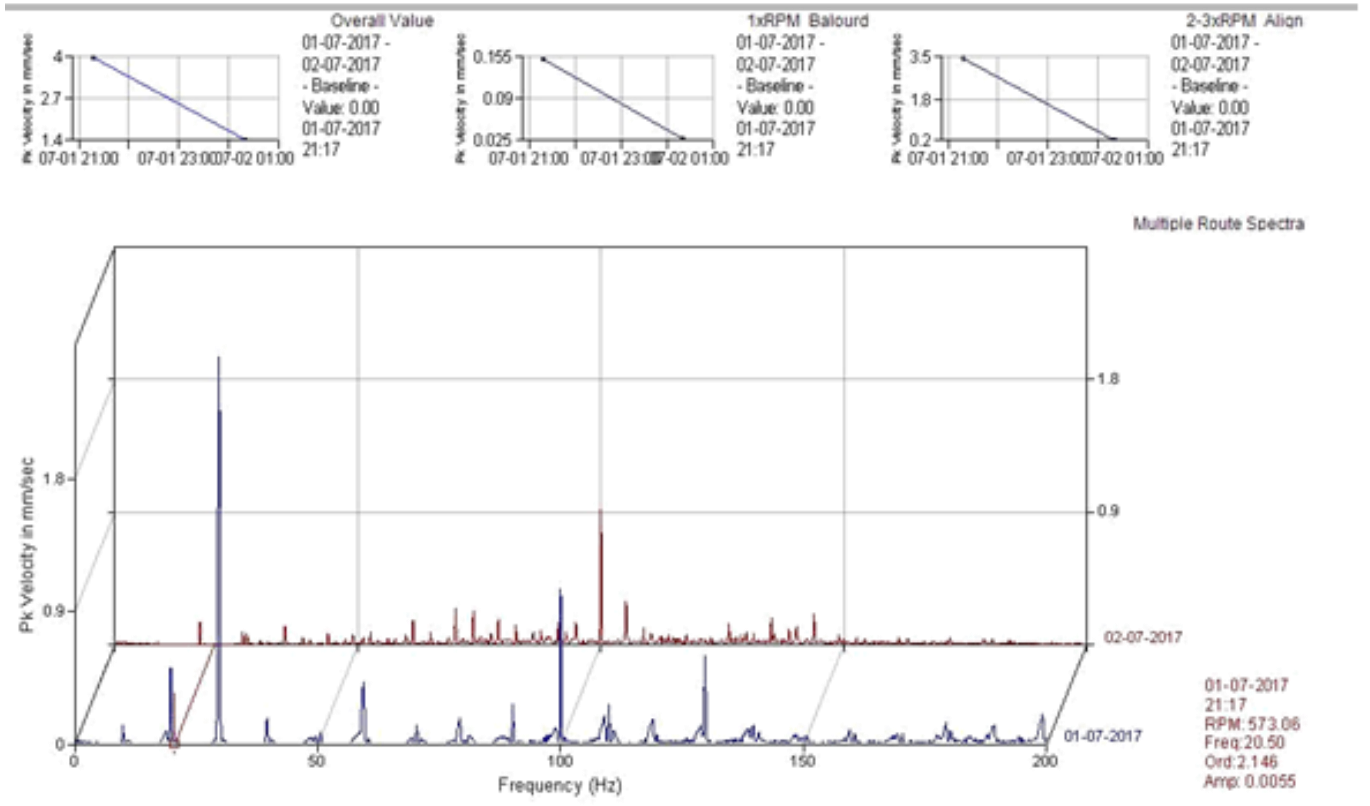

Fig. 15. Vibration spectra of motor outboard vertical 

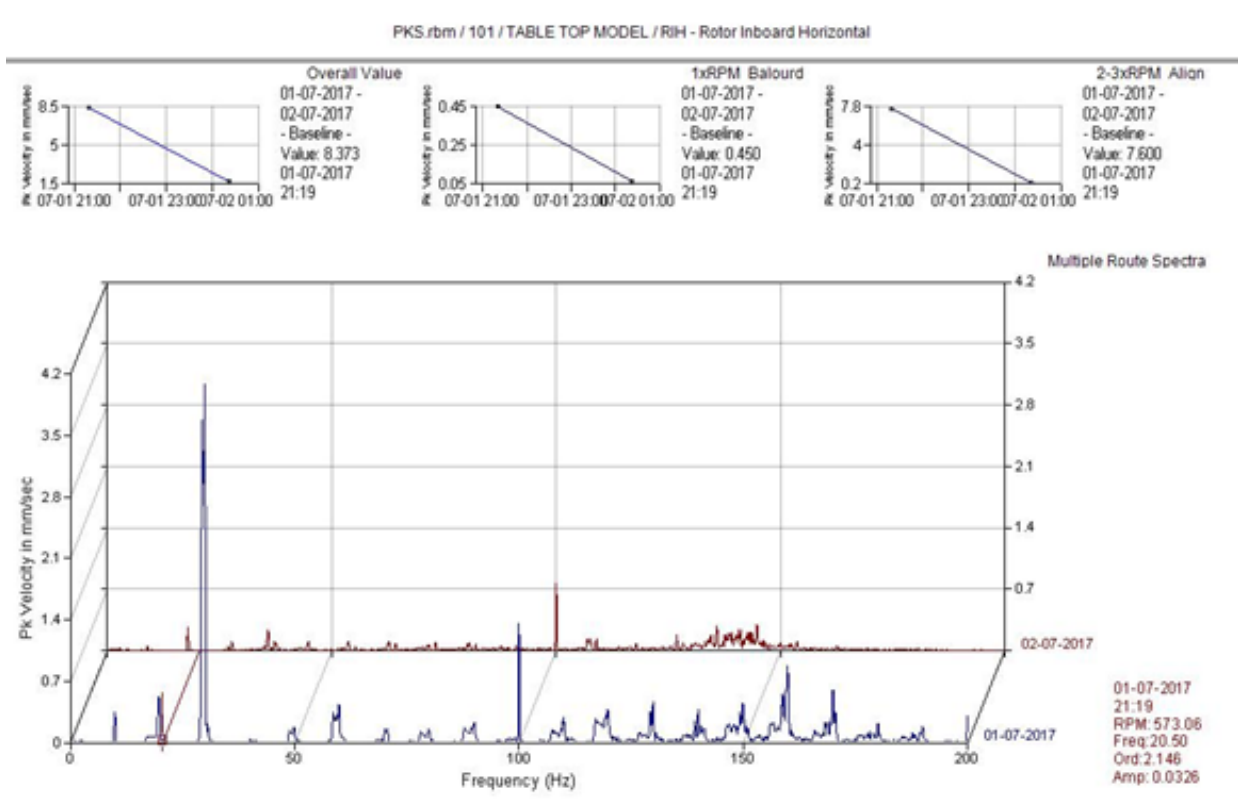

Fig. 16. Vibration spectra of rotor inboard horizontal

PKS TOm / 101/TABLE TCP MCDEL / RN - Rohor inboard Vertical
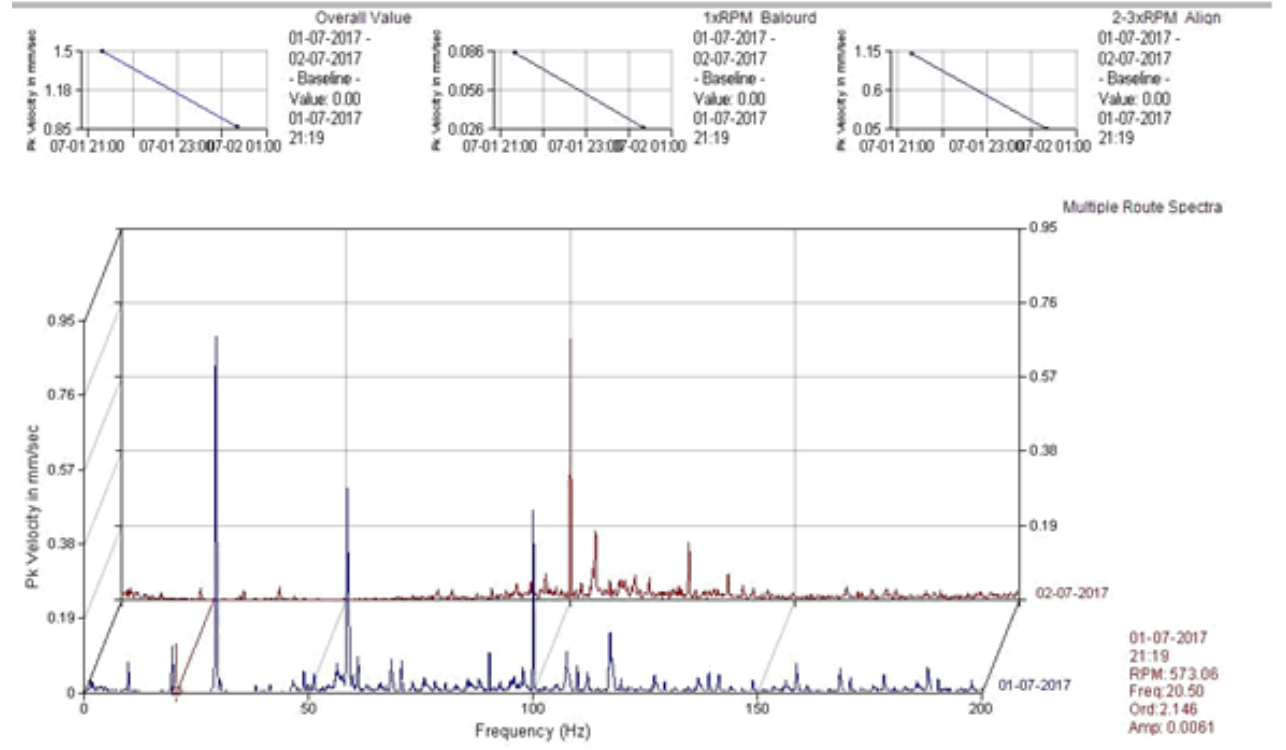

Fig. 17. Vibration spectra of rotor inboard vertical 
PKS ItOm / 101/ TABLE TOP MODEL / ROH - Rotor OUtboyro Horizontal
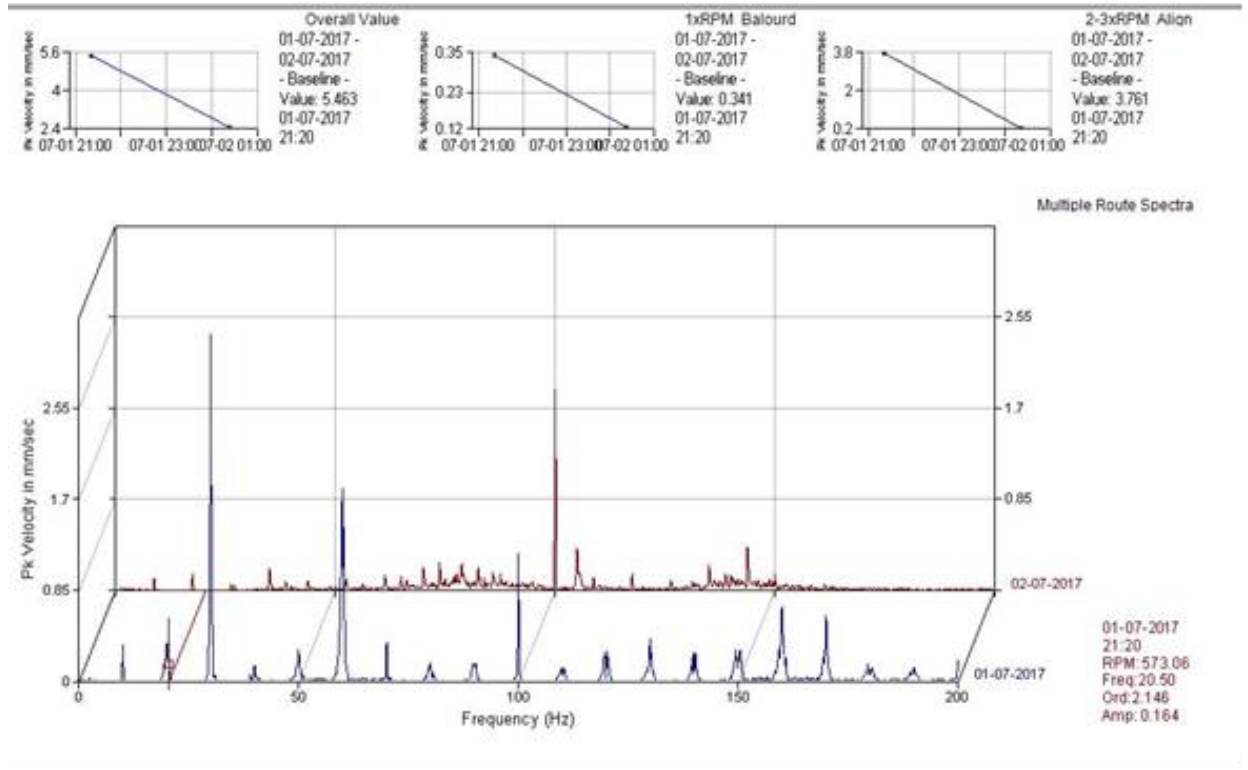

Fig. 18. Vibration spectra of rotor outboard horizontal
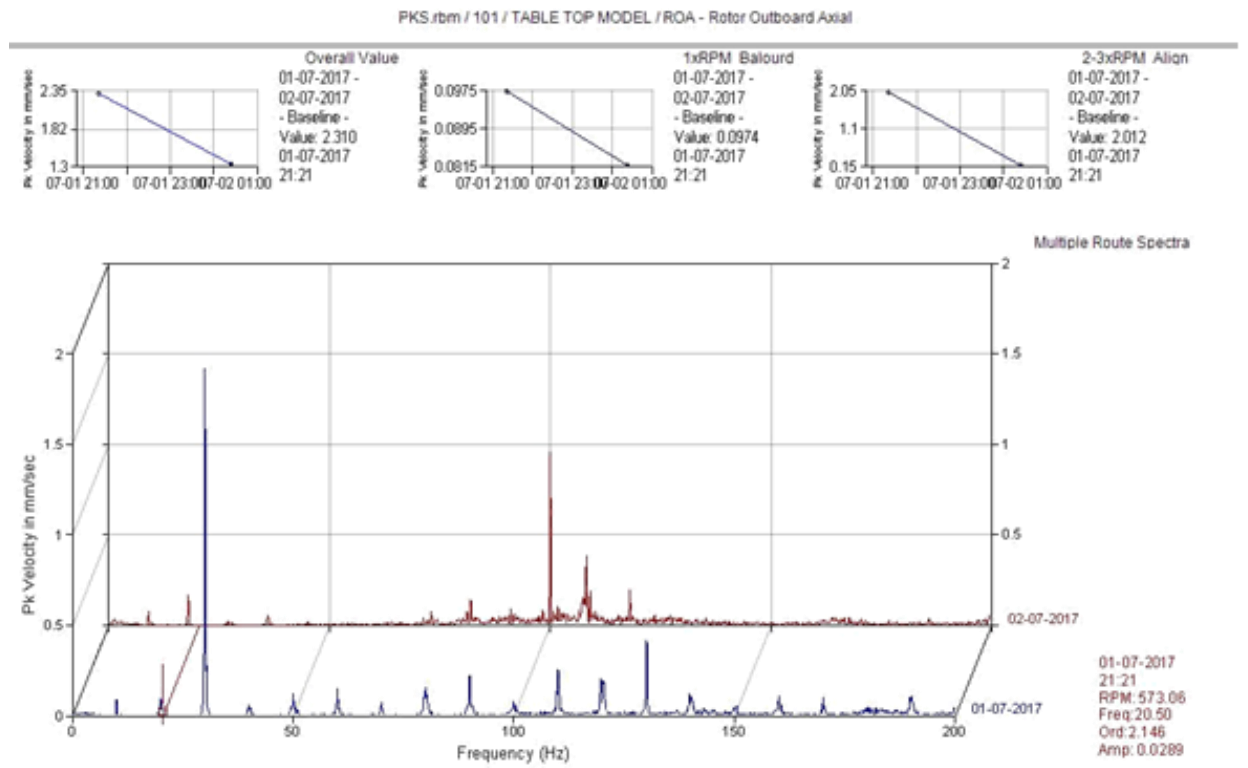

01.07 .201 2121 . 390 Freq 20.50 Drd 2.146 Amp: 0.0289

Fig. 19. Vibration spectra of rotor outboard axial 
PKS rom / 101/ TABLE TOP MOOEL / ROV - Rotor Outboard Vertica!
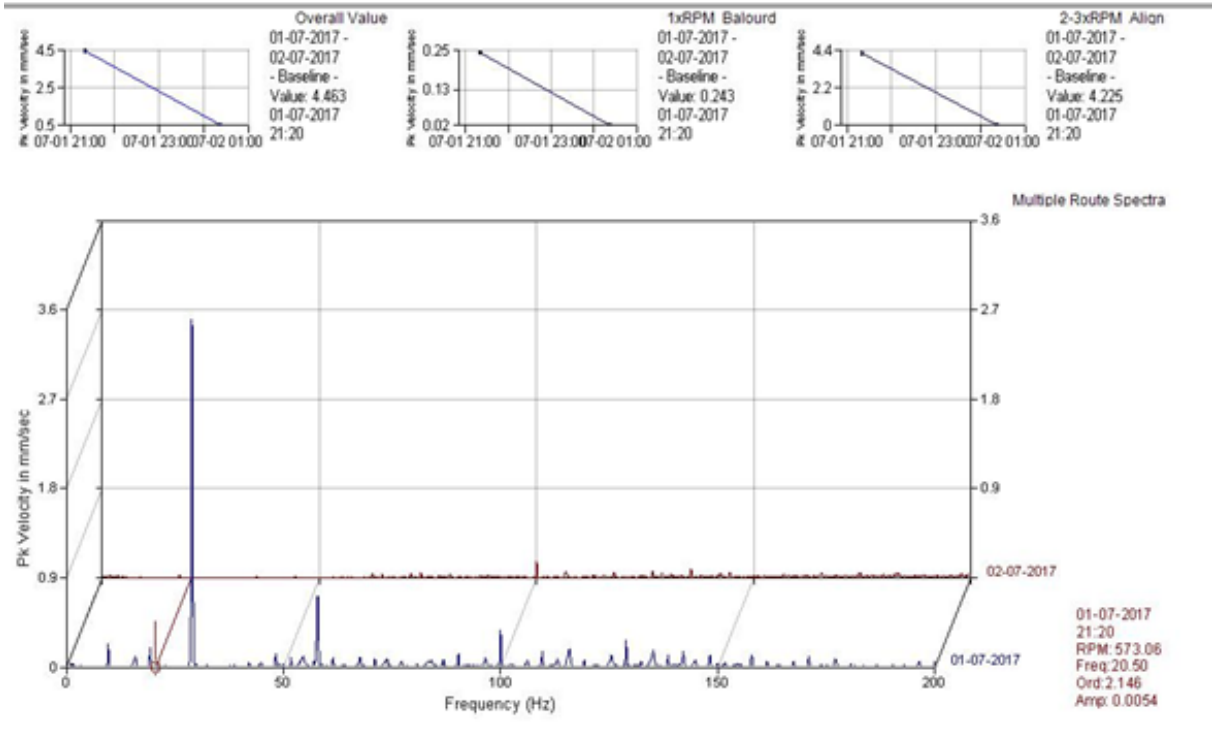

Fig. 20. Vibration spectra of rotor outboard vertical

5. As can be seen in above vibration spectra, $3 x$ peaks were quite prominent before any alignment was made, which resulted in suspecting there might be some misalignment with the 3 jaw coupling.

6. After the three jaw coupling was aligned properly, we can see, as indicated with vibration spectra in the foreground, the $3 \mathrm{x}$ peaks completely disappear, which shows that the problem originated in the coupling.

For Balancing:

1) The table top model was used for studying how to balance a rotating machine. As it was a perfectly balanced model, first some weight in the form of Nut \& bolt with washers were fitted to make the rotor unbalance. The same figure shows the reflective tape location to read the phase from the taco-probe. 


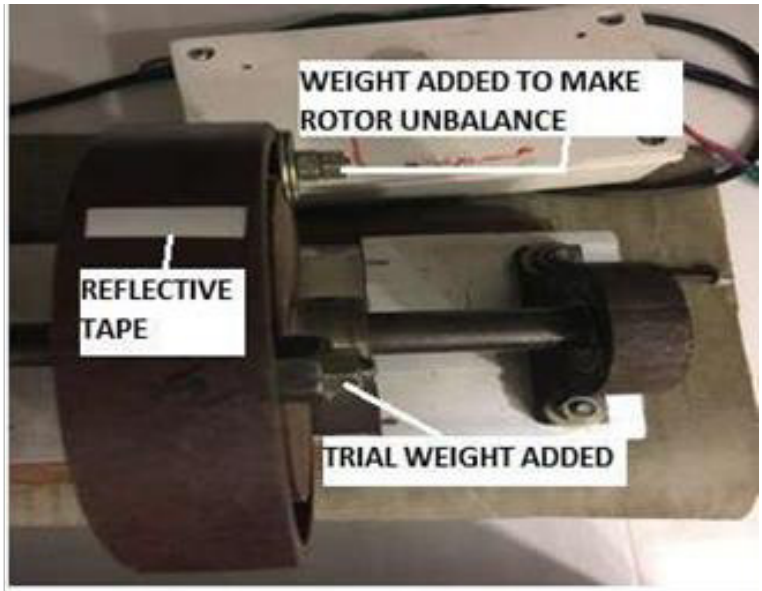

Fig. 21. Table top model to study unbalance

2) First a reference reading was taken with the CSI 2140 analyser in basic vibration mode, shows the reference run reading showing magnitude and phase was captured in the analyser.

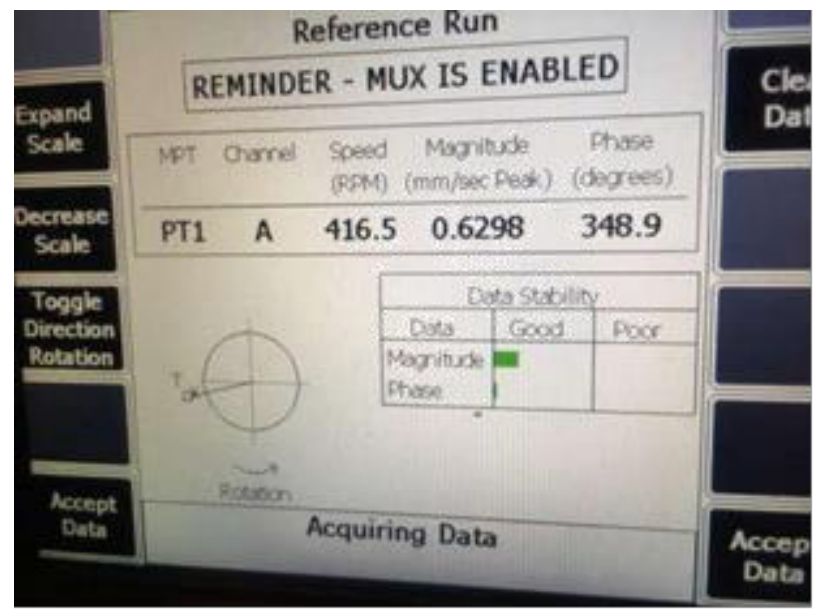

Fig. 22. Reference run taken for balancing

3) Next the trial weight was added to the rotor plane as shown in Fig 5.12 and another run was taken and data stored in the analyser 


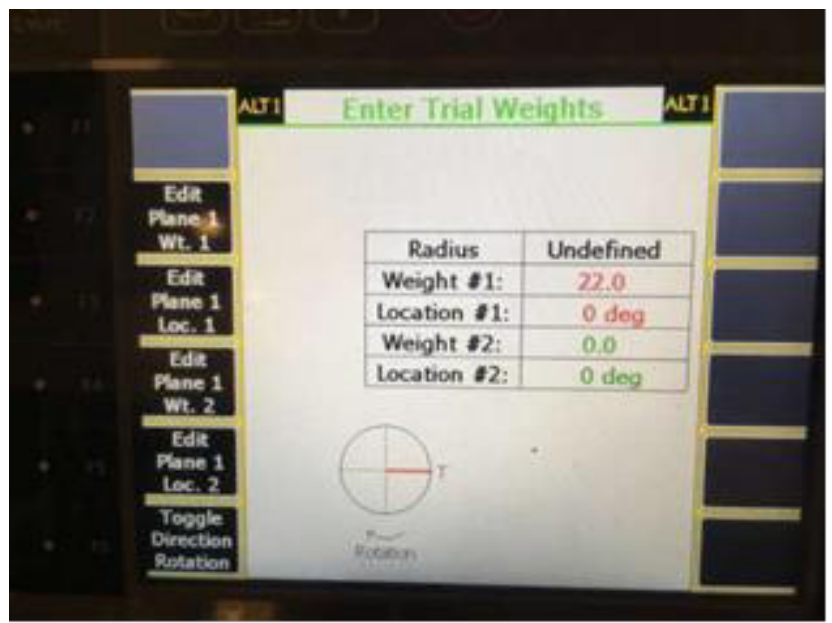

Fig. 23. Adding trial weight details to the analyser

4) Then the analyser with its in-built software calculates the amount of actual weight to be added or removed from the location to balance the machine.

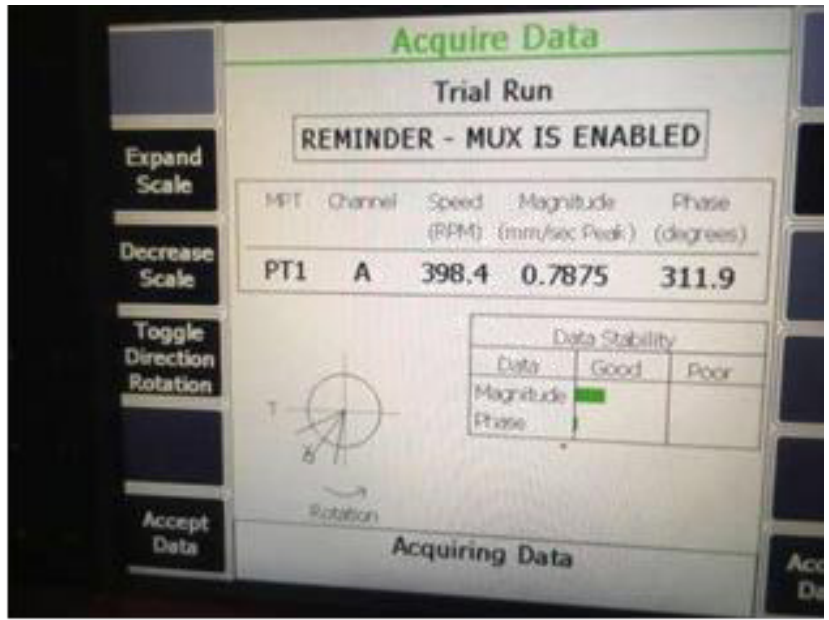

Fig. 24. Acquiring trial run data 


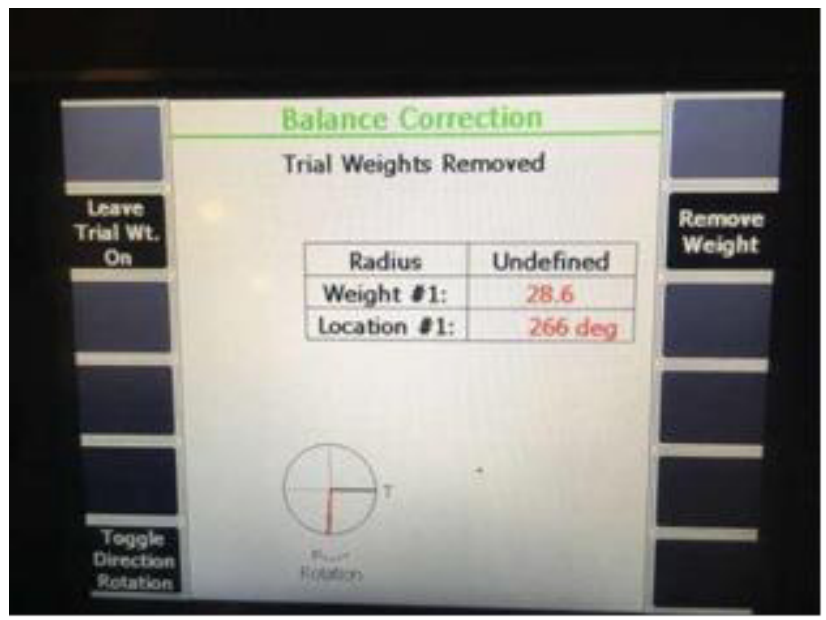

Fig. 25. Weight to be removed as calculated by the analyser

5) After removal of the weight as indicated by the analyser a run was taken, which shows the residual unbalance in the machine. To remove this further balancing has to be done, which is known as trim balancing with the repetition of same procedure.

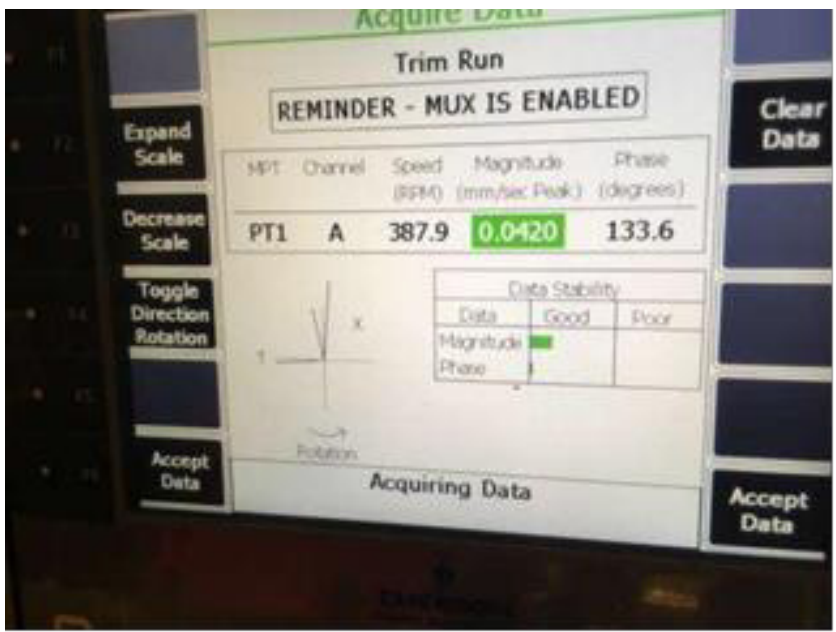

Fig. 26. Acquiring final trim run data 
6) At the end the analyser indicates the balance quality, which checks whether the machine is balanced to its tolerance level or not. If not we can still proceed for trim balancing until it achieves the tolerance level for the particular machine.

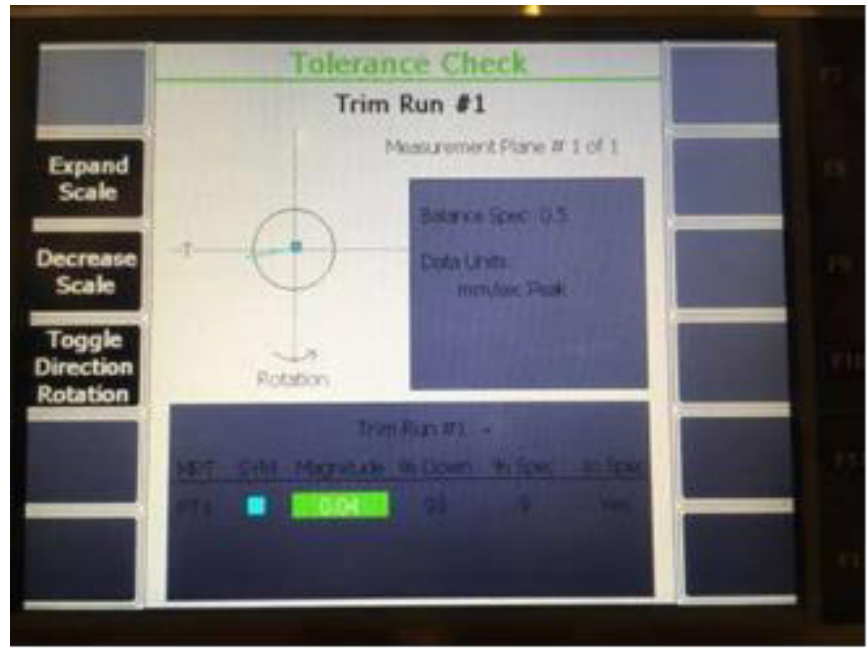

Fig. 27. Tolerance check for the balancing done

\section{Conclusions}

From the project, it can be concluded that, adapting the philosophy of predictive maintenance can be beneficial to all industries and plants. Using predictive maintenance, we can increase the life spans of various machinery and avoid unwanted shutdowns and downtime. Predicting a failure before it occurs can help analyze the root cause of the occurrence of machine fault and can prevent the failure of the machine in question which might in turn cause damage to other machines in the system. Vibration analysis plays a major role in predictive maintenance and as can be inferred from the experiments, defects that are not visible to the naked eye and that seemingly do not show any physical symptoms can be detected by this method, and thus is very important in the topic of plant maintenance.

\section{References}

1. Basic Vibration Analysis Course 2031, Educational Services, Machinery Health Management, EMERSON (2017)

2. Vibration training course book, Mobius (2012)

3. ISO 10816-3 Vibration Severity Chart. 